\title{
LO RACIONAL, LO RAZONABLE: DEL MODELO MATEMÁTICO Y EL FÍSICO A LA NATURALEZA HUMANA
}

\author{
ALEJANDRO PATIÑO ARANGO ${ }^{1}$
}

alejandro.patino@ucaldas.edu.co

Manizales, 2011-05-04 (Rev. 2011-09-12)

\section{RESUMEN}

En esta investigación pretendo desarrollar tres nociones: racional, razonable y textura abierta. Cada una a través de Descartes, Hume y Waisman, respectivamente. En el primeroaracional la identificamos con el método matemático; en el segundo, mostramos cómo la moral es razonable; en el tercero, pretendo mostrar cómo la moral y el derecho tienen las características tanto de lo razonable como de la textura abierta. Es decir, corresponden a saberes abiertos, en contraposición a los saberes cerrados como los de la lógica formal.

\section{PALABRAS CLAVE:}

Textura abierta, racional, razonable, modelo matemático, conjeturas, lógica formal, escepticismo.

\section{THE RATIONAL, THE REASONABLE: FROM THE MATHEMATICAL AND PHYSICAL MODEL TO HUMAN NATURE}

\section{ABSTRACT}

This research aims to develop three concepts: rational, reasonable and open texture through Descartes, Hume and Waisman's theories respectively. In the first one, we identify the rational with the mathematical method; in the second one, we show how moral is reasonable; and in the third one the aim is to show how moral and law have characteristics of both, reason and open texture. In other words, moral and law correspond to open knowledge in contrast to closed knowledge as those of formal logic.

\section{KEY WORDS:}

Open texture, rational, reasonable, mathematical model, conjectures, formal logics, skepticism.

Como se pueden dar cuenta, nos encontramos en esta investigación con tres términos que parecen ser usados de manera indiscriminada, pero realmente corresponden a términos distintos cuyos horizontes de aplicación son muy divergentes. De la misma manera que se presenta la división entre ciencias del espíritu y ciencias naturales: la racionalidad de un saber no es la misma racionalidad del otro. Cada uno posee sus propios criterios y principios de aplicación. Sin embargo, esto no significa que corresponda a dos facultades distintas en los seres humanos, simplemente es la misma facultad de la razón que realiza dos o más funciones distintas. No obstante, Kant estableció la distinción entre lo racional que le corresponde al entendimiento como a las ciencias empíricas y matemáticas y lo racional que le corresponde a la razón como la razón práctica propiamente dicha. Por ello, concibió tanto el entendimiento como la razón, facultades del conocimiento que tienen su 
horizonte de aplicación: tierra y mar. El entendimiento se apoya en la objetividad de lo condicionado y la razón se apoya en la subjetividad de lo incondicionado: lo racional y lo razonable respectivamente: razón pura y razón práctica. La razón pura tiene como la paloma el punto de apoyo, no siempre puede permanecer volando; en cambio, en la razón práctica la paloma se queda sin punto de apoyo y permanece volando eternamente, hasta el momento en que se encuentre la adhesión del interlocutor.

Realizo esta breve introducción con el fin de diferenciar desde ahora los dos caminos en que nos movemos y que, a pesar de que se pueden cruzar, se diferencian claramente. La falacia la cometemos cuando confundimos nuestra ruta o reducimos el uno al otro, seguramente llegamos al lugar equivocado, cometemos falacia naturalista como la llamó Moore. Razón por la cual trata de evitar esta falacia, mostrando cómo el camino de los valores se diferencia del camino de los hechos. A esto podemos agregar que cada camino tiene sus propios principios: Principia Ethica y Principia Mathematica para contrastar el título de dos escritos de filósofos contemporáneos: Moore y Russel. Ellos se dieron cuenta de esta diferencia. Sin embargo, podemos introducir una nueva falacia que pienso se puede denominar racionalista. Esta consiste en la pretensión, que claramente se encuentra desde Descartes en sus diferentes obras: falacia racionalista.

\title{
LA VERDAD Y LA VEROSIMILITUD
}

Para tratar estas dos nociones, debo recordar el siguiente texto en el Fedro de Platón:

\begin{abstract}
"¿Y vamos a dejar dormir a Tisias y a Gorgias, que vieron que había de estimarse más las verosimilitudes que las verdades, y por la fuerza de su palabra hacen aparecer las cosas pequeñas como grandes, las grandes como pequeñas, lo que es nuevo como si fuera viejo, y lo contrario como si fuera nuevo, y descubrieron cómo hablar con concisión o extenderse indefinidamente sobre cualquier materia?" (Platón, 1969, p. 360).
\end{abstract}

Como nos podemos dar Observemos que aquí lo que menos interesa es la realidad, sino hacer aparecer esta como no es. El problema se presenta en el lenguaje, a través de la palabra se invierte la realidad. Se debe notar que no es un problema de percepción, ya que se pueden tener percepciones equivocadas sin ser consciente de ello como sucedió con la teoría del geocentrismo, no era un problema del lenguaje o de la argumentación, simplemente era un problema de percepción y que se reducía a lo que se percibía, no se pensaba que los sentidos podían ser fuente de error como lo demostró Descartes cuando concibió la duda metódica. El problema de la percepción es muy diferente al problema del lenguaje. No era que Tisias y Gorgias cambiaran la realidad, esta permanecía tal como la percibían, el problema se centraba en mostrar a través del lenguaje que la realidad era lo contrario. Para Tisias y Gorgias no interesa la verdad, interesa la dialéctica, es decir, aquello que no es posible es probar o demostrar. De ahí que no se persiguen en este tipo de argumentación las inferencias necesarias o verificables. Al contrario, interesa aquello sobre lo cual se busca la adhesión del interlocutor. En la demostración necesaria se presenta la aceptación, en la argumentación se presenta la adhesión Es muy diferente la aceptación a la adhesión. En la primera, el sujeto simplemente cumple una función pasiva, en la argumentación la adhesión es activa. El sujeto simplemente se somete a 
los hechos, y los hechos y demostraciones se suceden sin que entren a mezclarse con los tics psicológicos de los individuos como sucede en la argumentación. Por esta razón, nos vemos en la obligación de afirmar que la argumentación es intersubjetiva, ella se da a nivel de la comunicación entre los seres humanos. De ahí que se mezclen el aspecto lógico con el psicológico, lo cual nos lleva a afirmar la presencia de la subjetividad. Esta es una actividad que se da entre los seres humanos. $Y$ digo esto, ya que no es posible hablar de argumentación entre los animales. Entre estos se puede hablar de comunicación, mas no de argumentación. Entre los seres humanos se habla de comunicación, pero mediante esta se puede realizar la argumentación.

Pero detengamos un momento en la definición clásica por género y especie que nace desde Aristóteles, y cómo la noción de sustancia predomina hasta la época moderna: "el hombre es un animal racional"2. Observemos que al afirmar la racionalidad, estamos dando cabida para afirmar su irracionalidad. Es decir, se afirma la racionalidad con el fin de mostrar que muchas veces y quizás muchas veces más nos comportamos de un modo irracional. Aquí no estamos haciendo referencia a la irracionalidad de los animales, que quizás la tomamos de un modo despectivo. Esto debido a la tendencia que tenemos de confundir la razón con el instinto, dice Brown: "Ser irracional es cosa que requiere considerable sofisticación [...] El hombre tiene la capacidad de razonar y de ser influido por la razón en forma en que no puede serlo, por ejemplo, un tigre hambriento" (Brown, 1965, p. 7). Al afirmar la sofisticación estamos diciendo que la irracionalidad a la que hacemos referencia es a la irracionalidad humana, excluyendo de esta manera a la animal que para nada tiene la sofisticación humana. Es por ello que el hombre mediante la razón posee la capacidad de influir en los demás, y cuando hablamos de influir estamos hablando que se tiene como fin buscar que el interlocutor cambie de opinión. Al realizar esta acción teleológica el interlocutor debe pasar de un estado psicológico a otro estado o a un estado diferente, ya que su fin es el cambio de opinión o que el interlocutor abandone su posición inicial para adherirse a la posición que tiene el locutor. Es en este momento donde se presenta la razonabilidad más que la racionalidad misma.

Observemos que la razón funciona de dos modos distintos, pero al fin y al cabo es la misma facultad de razón y no son dos facultades distintas.

Con base en la reflexión que estamos haciendo, debo decir que la matemática es racional y las acciones humanas son razonables y en caso específico la moral. De ahí se sigue que la moral y la política son razonables y que la argumentación es su instrumento de persuasión fundamental.

Tanto sobre lo racional y sobre lo razonable voy a tomar dos casos específicos de la filosofía como son el Método de Descartes y la Moral en Hume, para adentrarme a analizar posteriormente la noción de textura abierta. Para entender la noción de racional podemos recordar lo relacionado con el método en Descartes.

El problema del Método. Cuando se está en posesión de verdades de carácter general las dificultades se resuelven subsumiendo los casos particulares dentro de las verdades generales a que correspondan. Como se ve claramente en el silogismo. Pero cuando las verdades generales se derrumban, el silogismo deja de ser útil como muy bien lo hacen ver tanto Descartes como Bacon ${ }^{3}$. Además, el silogismo no permite alcanzar nuevas verdades, ya que la conclusión está contenida en las premisas, siendo una tautología. Afirmemos que en la época moderna se presenta la decadencia del silogismo ${ }^{4}$. 
Para la comprensión se debe tener presente tanto la influencia que se da en Descartes de las matemáticas que aprendió en su formación, es decir, la geometría de Euclides, como su propia geometría analítica ${ }^{5}$. Detengamos un momento en las cuatro reglas del método.

Primera Regla o Regla de la evidencia. En esta regla encontramos una doble faceta: la negativa que consiste en abandonar todo juicio que no esté fundado en la evidencia, ya que lo evidente se presenta después de andar el camino regresivo, haber eliminado los prejuicios. La positiva que consiste en asentir a lo claro y a lo distinto. Esta segunda etapa en el proceso seguido por Descartes viene después de la etapa negativa. Una vez hemos quitado el velo nos encontramos con lo claro y lo distinto. No olvidemos que los prejuicios o prevenciones son la diversidad de las opiniones que hemos aceptado sin haber sido examinados por la razón. De ahí que sean irracionales sin haber examinado su fundamento como también lo reafirmaDescartes en los Principios de la Filosofía (2004)(1). Al no ser examinados por la razón nunca podemos decidir sobre su verdad o falsedad. Los prejuicios nos engañan, aunque tengan apariencia de verdad. No son examinados por la razón, ya que son adquiridos a través de la costumbre, la opinión ajena y la inclinación natural. Eliminados los prejuicios, lo evidente se hace presente de un modo patente y claro, siendo aquello que se presenta de un modo inmediato al espíritu. Se da el paso del prejuicio a la intuición ${ }^{6}$.

Al prejuicio se opone la intuición, así en Reglas para la Dirección del Espíritu (en adelante RDE) define la intuición:

"Entiendo, no la creencia en el variable testimonio de los sentidos o en los juicios engañosos de la imaginación mala reguladora- sino en la concepción de un espíritu sano y atento, tan distinta y tan fácil que ninguna duda quede sobre lo conocido; o lo que es lo mismo, la concepción firme que nace en un espíritu sano y atento, por las luces naturales de la razón" (Descartes, 1903b, p. 277).

Al analizar esta definición de intuición, nos damos cuenta de que no es una intuición sensible a la manera como la concibe Kant en la estética trascendental: el espacio y el tiempo son intuiciones a priori de la facultad de la sensibilidad. Son percepciones claras y distintas que se presentan al espíritu. Claras en el sentido de que las percibimos de un modo evidente y sin ninguna confusión ante la mente y distintas en cuanto no contienen nada obscuro. La intuición siempre está referida a lo verdadero y de ningún modo admite duda, es lo axiomático. La percepción de lo presente está acompañada del asentimiento y no puede darse sin él. Por ello, el método es el camino que nos conduce a la mostración de lo verdadero. Hemos afirmado, que en esta primera regla del método se presenta el paso del prejuicio a la intuición y en este momento no puede ser engañado por ninguna clase de experiencia, dice el filósofo francés: "El entendimiento no puede engañarse en ninguna experiencia si se limita a la intuición precisa del objeto tal como lo posee en sí mismo o en la imaginación" (Descartes, 1903b, p. 315).

Segunda Regla o Regla del análisis o de la división. Hay que analizar lo que Descartes llama cuestiones $^{7}$; esta regla consiste en dividir lo complejo en lo simple, mejor, dividir las naturalezas complejas en naturalezas simples. Es decir, las proposiciones simples contienen ideas claras y distintas aprehendidas por la intuición, permitiendo decidir sobre su verdad o falsedad. De ahí que su geometría sea analítica.

Observemos que el procedimiento del análisis no solo es aplicable a las matemáticas, sino que también se aplica a la metafísica como sucede con 
el ejemplo de la cera que Descartes analiza en la Meditación II. Debo destacar que a partir de este ejemplo llega a analizar el "yo".

En resumen, destaquemos que el análisis se le puede aplicar a: la matemática, a la geometría, a la física, a la metafísica como sucede con el análisis de la palabra "yo", no olvidemos la expresión: "Divide y vencerás".

Pensando en la lógica matemática correspondería quizás a la descomposición de las proposiciones moleculares en las proposiciones atómicas, como bien lo exponen tanto Russell como Wittgenstein. En esto consiste un análisis perfecto.

Tercera Regla o Regla de la síntesis o composición. Es menester otro procedimiento paralelo a la división y de sentido inverso, ya que no podemos permanecer solo en lo simple. Es el procedimiento de la deducción, el cual nos conduce a proposiciones complejas-verdaderas a partir de las nociones simples; se trata de un tránsito de lo simple a lo complejo. Digamos que no se trata de seguir el orden lógico por género y especie, en el sentido como lo concibió Aristóteles y lo desarrolló Porfirio ${ }^{8}$, sino la concatenación de la dependencia y la fundamentación del conocimiento. Consiste en derivar lo complejo a partir de lo simple, es decir, se puede tener conocimiento de lo complejo, sin ser evidente por sí misma, dice: "Antes de contestar a la pregunta, definamos la deducción. Consiste en una operación por la cual comprendemos todas las cosas que son consecuencia necesaria de otras conocidas por nosotros con toda certeza" (Descartes, 1903b, p. 278). Es claro que en la deducción se presenta un movimiento y cierta sucesión necesaria, movimiento que no se presenta en la intuición ${ }^{9}$.

Digamos que se ha dividido el conocimiento en proposiciones simples y en cuestiones. De esta manera el tercer precepto supone el segundo. A la descomposición de las cuestiones en ideas simples sigue la composición o la recomposición sintética de un sistema de proposiciones, según una clara dependencia.

Todas las verdades se siguen unas a otras en el reino de la razón, se encuentran atadas unas a otras como los eslabones de una cadena. Recordemos que no es una deducción silogística. Debo destacar los siguientes aspectos de la deducción:

1. La deducción puede verse como un procedimiento de composición.

2. La deducción amplía el conocimiento intuitivo, y en el fondo se basa en la intuición, pero entre intuición e intuición se presenta la derivabilidad necesaria y la memoria.

3. La intuición es estática, en la deducción se presenta un movimiento, es una mirada intelectiva en la que se pasa de una proposición a otra, dice: "De este modo el anillo de una larga cadena está unido al primero -aunque no podamos abrazar con una sola ojeada todos los anillos intermediarios que los une" (Descartes, 1903b, p. 278-279).

4. La deducción es una sumatoria de intuiciones en acción, la deducción se reduce a intuiciones, dice:

"Al primer golpe de vista no podemos distinguir los anillos de una cadena muy larga: sin embargo, si vemos la unión de cada anillo con el que le precede y con el que sigue, tendremos derecho a afirmar que hemos visto cómo el último se enlaza con el primero" (Descartes, 1903b, VII, p. 292). 
Por último, el cuarto precepto es el de la enumeración, es el vigilante de la memoria; obliga a repasar toda la cadena en un nuevo movimiento rápido de intuición, de tal manera que hay que reconocer la cadena entera con un movimiento continuo como muy bien lo desarrolla en la regla VII (Descartes, 1903b, VII, p. 292).

En síntesis, el método es el camino hacía el conocimiento verdadero, es decir, es la búsqueda de la verificación universal, es un modelo racional universal en el que no está incluido la retórica. Sin embargo, no olvidemos que a pesar de las críticas que Descartes le hace a la retórica se ve en la obligación de introducir en la parte III del Discurso del método una moral provisional, para ello introduce cuatro máximas que tienen que ver más con lo razonable como sucede con las tres primeras y la cuarta máxima que tiene que ver con lo racional, dice Descartes:

"Considerando constantemente la limitación impuesta a nuestra débil naturaleza, se persuadieron que únicamente nuestros pensamientos estaban dentro del poder de nuestras mezquinas facultades, $y$, por consiguiente que ninguna afección debían inspirarnos las cosas, puesto que nada era nuestro, fuera de los pensamientos" (Descartes, 1903a, p. 18).

\section{MORAL COMO RAZONABLE EN HUME}

Empiezo, entonces, observando cómo Hume evita llegar a un egoísmo al plantear la objetividad universal de los juicios morales. Con base en este presupuesto, concibe la diferencia entre "es" y "debe", diferencia que le permite argumentar la autonomía de la moral como la va a concebir Hare en el siglo XX. Pienso que esta diferencia conduce al filósofo escocés a criticar a quienes habían ignorado tal autonomía, concentrando sus reflexiones en el "es" o camino de la metafísica. Esto los lleva a adoptar una especie de reduccionismo descriptivo, y que Hume crítica al hablar de "los usos falaces del lenguaje", y que Austin denomina "Falacia descriptiva" cuando se confunden expresiones como: "Escribir" y "Prometer". Según Moore (1997)reducen el significado del término "bueno" a hechos naturales como aquello que produce placer, teoría sostenida por los naturalistas y que, según Moore, en su Principia Ethica cometen "falacia naturalista"10.

Para ello Hume concentró la reflexión en el acto de habla de "prometer", ya que si "prometo" entonces "debo". Pienso que en la concepción de Hume (1977)en "Del carácter obligatorio de las promesas", se encuentran conceptos fundamentales que serán desarrollados posteriormente por Austin y Searle. Conceptos como: intención, convención, las promesas no son verdaderas ni falsas, el criterio de las promesas es lo laudable o lo no laudable, la importancia de la expresión "yo prometo", el acto de prometer como institución social. Antes es necesario observar que el capítulo de Hume sobre las promesas es poco conocido por los filósofos analíticos, a pesar de que el escrito sobre el paso del "es" al "debe" se hubiera convertido en un tema clásico de reflexión. Mejor analizan el acto de habla de prometer, mas no se refieren en este tema a Hume.

Hume es claro en sostener que la moralidad no consiste en una conformidad con la razón como sucede con las demostraciones matemáticas, en las cuales se presenta una eterna adecuación a partir de la yuxtaposición y comparación de ideas. Por ello Hume busca otros principios que permiten diferenciar el bien y mal morales y no se queda en la razón como había sucedido tradicionalmente ${ }^{11}$. La razón no puede 
influir en la moralidad, ni puede derivarse de esta, en la moralidad no es posible hablar de causa-efecto como sucede en las ciencias. La moralidad conserva de modo constante su propia autonomía. Es decir, una facultad inactiva no puede influir en una facultad activa. De esta manera las reglas de la moralidad no pueden derivarse de la razón. Sin embargo, debemos decir que el principio de moralidad es un principio universal, que está presente en todos los hombres, como bien los demuestra Hume (2003) en Investigación sobre la moral: depende de una especie de sentimiento interno que la naturaleza ha hecho universal. Una cosa es el sentimiento de universalidad y otra cosa es la acción particular de cada individuo. Lo que le pone el sello de moralidad a cada acción es la universalidad, dada por una especie de razón superior que poseen los seres humanos. Dice:

"La única diferencia estaría en que nuestra superior razón pueda servirnos para descubrir el vicio o la virtud, aumentando así la censura o alabanza, pero este descubrimiento supone estas distinciones morales en un ser separado: un ser que depende sólo de la voluntad y del apetito, y que tanto en el pensamiento como en la realidad tendría que poder considerarse como algo distinto de la razón" (Hume, 1977, p. 688).

Según el texto, este argumento, que en el fondo tiene que ver con que la moralidad no funciona como las matemáticas, es reconfirmado cuando Hume concibe que tampoco tenga que ver con las cuestiones de hecho como percibir una mesa o realizar un experimento en el laboratorio. Esto se sigue claramente del ejemplo que trae el filósofo relacionado con el asesinato. El vicio no está en el hecho mismo, sino que está en el sujeto producido por el principio universal que mencionamos antes ${ }^{12}$. De esta manera mientras nos dediquemos a analizar el hecho mismo, el valor moral se nos escapara completamente. El valor moral solo se puede descubrir por el sentimiento como lo sostiene tanto en el Tratado (1977)como en la Investigación(2003). En el Tratado habla de nuestro propio pecho y en la Investigación habla del corazón. Podríamos decir que razón superior, nuestro propio pecho, sentimiento y corazón tienen la misma significación en ambas obras. Esto para indicar que la valoración no está en los hechos, sino que la encontramos en nosotros mismos. Sin embargo, debemos tener en cuenta que esta es otra especie de cuestión de hecho, pero que se encuentra en nosotros mismos y que es más sentida que pensada, dice: "He aquí una cuestión de hecho: pero es objeto del sentimiento no de la razón" (Hume, 1977, p. 689). Estos análisis llevaron a Hume a establecer la distinción entre hecho y valor, expresada mejor como la distinción entre "es" y el "debe" que el filósofo mismo expresa claramente y que ha sido objeto de mucho derrame de tinta en la filosofía contemporánea ${ }^{13}$. Hume mismo habla de las proposiciones de "es" que no están conectadas con el "debe". Esto le permite introducir esa especie de sentimiento natural, que equivale a una especie de razón en estado natural o principio universal del sentimiento moral como el mismo lo muestra en el primer apéndice de la Investigación sobre la moral ${ }^{14}$, ya que solo de los sentimientos del espectador surge la belleza y la elegancia. Sentimiento que tiene la característica de universalidad, ya que no se refiere a ninguna experiencia particular. Veamos el siguiente texto: "Sólo cuando un carácter es considerado general y sin referencia a nuestro interés particular causa esa sensación o sentimiento en virtud del cual lo denominamos moralmente bueno o malo" (Hume, 1977, p. 694). Universalidad que Hume sostiene en varias partes de la obra. Es decir, si consideramos determinada acción como buena, esta misma acción realizada por otra persona necesariamente debe ser considerada como buena siempre y cuando se encuentre en las mismas circunstancias que la persona anterior. Entonces, necesariamente tenemos que ser consecuentes con ese principio de universalidad. A no ser que las circunstancias cambien como lo observa el mismo filósofo. 
Por otro lado, para aclarar el criterio de verdad de los juicios morales en Hume, debemos afirmar que el criterio de valoración de las demostraciones matemáticas es el de verdadero, el del conocimiento del mundo empírico es el de verdadero falso, y el de la moralidad es el de laudable, razonable, afortunado. Es decir, por un lado se dan los criterios de racionalidad, por otro se presentan los criterios de razonabilidad. Con base en estos criterios entra la comprensión de la moralidad que el mismo Hume intenta fundamentar. Ahora bien, es claro que esta comprensión es una comprensión pragmática, mas no lógica. Comprendemos el paso "es""debe" de un modo pragmático. Pero este paso o salto no es lógico. Surge, entonces, una especie de razonabilidad pragmática de la moral ${ }^{15}$. Hume mostró cómo la verdad se aplica a las matemáticas necesariamente y a las ciencias empíricas de manera probable.

Observen que coloco el énfasis en la expresión razonabilidad pragmática, en este momento el hombre es un individuo de carne y hueso como lo concibió Unamuno y no es un hombre calculador y frío. No es dogmático y admite la contradicción.

Para terminar, voy a recordar dos textos que nos expresan de modo claro la diferencia entre el individuo máquina o calculador o regido por las leyes eternas o de la divinidad y el hombre del la vida cotidiana impregnado con todas sus pasiones, con toda su moral y política. Leo primero un texto de Hume:

\begin{abstract}
"¿Dónde estoy, o qué soy? ¿A qué causas debo mi existencia y a qué condición retornaré? ¿Qué seres me rodean; sobre cuál tengo influencia, o cuál la tiene sobre mí? Todas estas preguntas me confunden, y comienzo a verme en la condición más deplorable que imaginarse pueda, privado absolutamente del uso de mis miembros y facultades" (Hume, 1977, p. 421).
\end{abstract}

\title{
La noción de textura abierta
}

Para adentrarnos a esta noción, desarrollemos primero la noción de Hart y luego la concepción que tuvo Waisman del mismo concepto, esto nos permitirá mostrar con algún detalle la influencia del filósofo en el jurista. La noción de la textura abierta del derecho la desarrolla Hart (2004) en el Capítulo VII que denominó "Formalismo y Escepticismo ante las reglas".

Hart parte de la idea de que en cualquier sociedad, el principal instrumento de control social esencialmente tiene la característica de ser general y no particular; las normas no se hacen para ser aplicadas a un individuo. Por ello las normas se hacen para ser dirigidas a clases de personas y a clases de actos y jamás son directivas particulares para cada individuo. Se da un predominio de lo general, ya que sería imposible la existencia de la sociedad si cada individuo tuviera sus propias normas o su propio sistema jurídico, de la misma manera que es imposible que para la denominación de los objetos externos cada objeto tuviese su propio nombre particular como muy bien lo concibió Locke cuando advertía sobre la imposibilidad de que cada grano de arena del mar tuviese su propio nombre, ya que estos nombres serían infinitos. Idea que llevó a Borges a escribir Funes el memorioso.

Hart explica los recursos que se han utilizado para comunicar las pautas generales: la legislación y el precedente. La legislación tiene mayor extensión, el precedente tiene menor extensión; de la legislación se hace un uso máximo, del precedente se hace un uso mínimo. 
Para entender la diferencia entre estas dos situaciones Hart trae dos ejemplos. El primero consiste en que un padre dice a su hijo: "Todos los hombres y niños varones deben descubrirse al entrar a la iglesia"; el segundo consiste en que otro padre descubriéndose la cabeza al entrar a la iglesia dice a su hijo: "Mira esto es lo que debe hacerse en estas ocasiones". En el primer ejemplo, se da la comunicación a través del lenguaje; en el segundo ejemplo, se da la comunicación a través del ejemplo. En esta segunda situación, surgen muchas inquietudes, a pesar de que vaya acompañada de muchas expresiones como: 'haz lo que yo hago', inquietudes que plantean muchas posibilidades como: ¿Hasta dónde debo imitar a mi padre?, ¿tiene importancia que nos quitemos el sombrero con la mano izquierda, en vez de hacerlo con la mano derecha?, ¿que lo hagamos lenta o rápidamente?, ¿en qué tiene que parecerse mi conducta a la suya para ser correcta?, ¿qué parte de su conducta ha de ser mi guía? Todo este tipo de inquietudes hacen que la comunicación por el ejemplo deje abierta muchas posibilidades. En esta situación se tendría que adivinar la intención del padre, lo que daría lugar a la especulación sobre los diferentes aspectos para que su conducta se pareciese a la del padre. Pero no sucede lo mismo en la situación de la comunicación verbal, ya que la expresión aparece como la fórmula verbal a seguir, rige el comportamiento subsiguiente de un modo claro que no da lugar a confusión o a inquietudes. Solo se tiene que reconocer situaciones concretas en las que se dé lugar a la aplicación de la fórmula general, sin dar rienda suelta a multiplicidad de alternativas, como lo dice Hart, es una simple conclusión silogística, solo se tiene que identificar los casos particulares para la aplicación de la norma general, dice Hart:

"Buena parte de la teoría jurídica de este siglo ha consistido en hacerse progresivamente cargo ( $\mathrm{y}$ a veces en exagerar) el hecho importante de que la distinción entre la falta de certeza de comunicación mediante el ejemplo dotado de autoridad (precedente) y la certeza de comunicación mediante el lenguaje general dotado de autoridad (legislación), es mucho menos firme que lo que sugiere el contraste ingenuo. Aun cuando se usen reglas generales verbalmente formuladas, en los casos concretos particulares pueden surgir dudas sobre cuáles son las formas de conducta exigidas por ellas" (Hart, 2004, p. 157).

Muchas veces las situaciones particulares no hacen parte de la regla general, ni en sí mismas se encuentran rotuladas a priori, ni tampoco la regla puede reivindicar los casos particulares. Esto no puede ser posible ni introduciendo las famosas hipótesis ad hoc, es decir, no podemos introducir una expresión que nos lleve a salvar, a la fuerza, la teoría; es el caso del color de los cisnes, en la situación de que encontremos un cisne negro, no es posible afirmar que 'este animal de color negro no es un cisne'.

Lo anterior significa que hay muchos casos particulares en el campo jurídico que no pueden ser subsumidos por la norma o no pueden ser deducidos de la inferencia silogística, dice Hart: "En todos los campos de experiencia, no sólo en el de las reglas, hay un límite, inherente en la naturaleza del lenguaje, a la orientación que el lenguaje general pueda proporcionar" (Hart, 2004, p. 157). Lo que trata de indicar es el límite que se da en los términos generales, los cuales se pueden aplicar a casos particulares que son subsumidos por el término general, pero hay casos particulares en los que se presenta la duda de la posible aplicación del término general, o casos que evidentemente no son posibles agruparlos dentro del grupo o clase o género como lo mencionamos antes cuando criticamos la definición aristotélica por género y diferencia. Sin embargo, cuando se presenta un caso particular, se tiene la posibilidad de acudir a la interpretación con el propósito de identificar el caso particular, de tal 
manera que según las características similares con otros individuos que caen dentro de la misma clase, puedan ser clasificados dentro de ella, dice Hart:

\begin{abstract}
"Estos últimos casos son situaciones de hecho, que la naturaleza o la inventiva humana continuamente presentan, y que sólo exhiben algunas características del caso obvio, mientras que le faltan otras. Los cánones de 'interpretación' no pueden eliminar aunque sí disminuir, estas incertidumbres; porque estos cánones son a su vez reglas generales para el uso del lenguaje, y emplean términos generales que también requieren interpretación" (Hart, 2004, p. 158).
\end{abstract}

Corresponde a casos particulares de los cuales no tenemos seguridad si los podemos incluir dentro de la clase general, a pesar de que posean ciertas características similares con otros casos anteriores, y posean otras características que no son similares. Cuando nos encontramos en esta situación, Hart propone como auxilio acudir a los cánones de interpretación, los cuales no pueden ser caprichosos ni subjetivos, sino que a la vez están sometidos a reglas que nos ayudarán en el uso del lenguaje cuando se aplica el término general al caso particular.

Pero cuando los casos particulares son evidentes y se pueden agrupar dentro del término general, no necesitamos interpretación y el reconocimiento de los casos particulares se hace patente y sin ninguna duda, se realiza esta identificación de manera automática y mecánica, estos casos son los casos que se presentan y se han presentado con frecuencia y de manera repetitiva, de la misma manera que no dudamos en clasificar una bicicleta como un vehículo. Aquí no necesitamos auxiliarnos de ninguna interpretación, a no ser que se quiera complicar lo evidente, o demostrar los axiomas o los principios que no necesitan demostración como la regla del reconocimiento, lo evidente no necesita demostración.

Cuando se presentan situaciones particulares confusas y de difícil identificación, quien realiza la interpretación debe guiarse por un acto de elección entre distintas alternativas o alternativas abiertas, dice Hart: "EI ámbito discrecional que le deja el lenguaje puede ser muy amplio; de modo que si la conclusión puede no ser arbitraria o irracional, es, en realidad una elección" (Hart, 2004, p. 159). Hay dos varios aspectos que podemos destacar del texto como: por una lado, que la discrecionalidad está dada por el lenguaje, ya que el lenguaje puede ser demasiado vago; y por otro, que la decisión final no puede ser arbitraria, es fruto de una elección que tiene que ser razonable.

Ahora podemos preguntarnos: ¿Cuándo surge la textura abierta? Esta se presenta cuando en los casos ordinarios se duda de las pautas de aplicación, bien sea la legislación o los precedentes. Cuando estas técnicas resultan indeterminadas en la aplicación o cuando dudamos de su aplicación a los casos particulares, nos encontramos en el campo de la textura abierta, dice Hart:

"Hasta aquí hemos presentado esto, en el caso de la legislación, como una característica general del lenguaje humano; la falta de certeza en el caso marginal es el precio que hay que pagar por el uso de términos clasificatorios generales de cualquier forma de comunicación relativa a cuestiones de hecho" (Hart, 2004, p. 159). 
Esto nos muestra que el lenguaje jurídico hace parte del lenguaje natural, del cual no se puede soslayar o esconder, y que la textura abierta está determinada más por el lenguaje natural que por el mismo lenguaje jurídico. Además, como lo explicamos en párrafos anteriores, el lenguaje jurídico funciona dentro de un contexto y dentro de un lenguaje natural que usamos para poder construir las distintas acciones lingüísticas: el lenguaje jurídico depende del lenguaje natural.

El lenguaje natural no es un lenguaje que funciona como las inferencias lógicas, como una máquina construida por el ser humano; las características del lenguaje natural permiten usar los términos generales para hacer mención a todas las acciones humanas; nuestras acciones tampoco pueden ser descriptas de manera precisa o perfecta, por una razón de gran importancia, aunque aparentemente simple: no somos dioses, somos hombres. Por ello, es imposible poseer un conocimiento completo y total de todos los hechos que existen en el mundo, somos ignorantes de la gran mayoría de los hechos, somos seres muy limitados y no poseemos el don de la omnisciencia, ya que el mundo está constituido por un número infinito de hechos naturales y de acciones humanas que es imposible encajar dentro de las categorías esquemáticas que nos brinda la ciencia. Es por ello que tanto el individuo en su cotidianidad como el jurista en su ejercicio, se ven en la obligación de elegir la interpretación de las acciones, dice Hart:

"Si el mundo en que vivimos estuviera caracterizado únicamente por un número finito de notas y éstas, junto todos los modos en que pudieran combinarse, fueran conocidas por nosotros, podríamos formular provisiones por adelantado para toda posibilidad. Podríamos elaborar reglas cuya aplicación a los casos particulares nunca exigiera una nueva aplicación. Todo podría ser conocido y, por ello mismo, la regla podría especificar por adelantado la solución para todos los problemas. Este sería un mundo adecuado para la teoría jurídica 'mecánica'” (Hart, 2004, p. 160).

Pero ese mundo es un ideal que apenas tiene existencia en nuestra mente, estaríamos en el determinismo, sería un mundo que no tiene realidad, solo podemos pensar que en un mundo así no necesitaríamos nada ni la ciencia. No tendríamos pasiones ni tendríamos que preocuparnos por el comportamiento, sería un mundo aburrido, mucho menos necesitaríamos juristas. Pero ese mundo no nos pertenece, no es el nuestro, no somos dioses. Para los juristas es imposible tener conocimiento de todas las acciones humanas, les es imposible a los legisladores humanos tener conocimiento de todas las posibles combinaciones de acciones que el futuro pueda deparar, ya que son simplemente legisladores humanos.

Como seres humanos no es posible que toda nuestra conducta sea mecánica, muchas veces realizamos comportamientos impredecibles para cualquier ser humano, inclusive para el legislador más sabio, ya que no puede tener conocimiento de todos los hechos. Los legisladores son seres humanos, ellos no pueden tener un conocimiento total de las acciones humanas. Por ello, se pueden presentar, con frecuencia, muchas situaciones que no habían sido contempladas por las normas, momento en el cual se necesita acudir al canon de la interpretación, al cual le es inherente la elección para la resolución del caso o de la situación particular de manera satisfactoria. Si el orden jurídico fuese un orden mecánico se caería en el vicio del formalismo jurídico, bastaría aplicar las normas sin tener en cuenta el contexto, ni la pragmática jurídica, no se tendría necesidad de interpretación ni de elección. Todas las acciones de los seres humanos estarían predeterminadas, este es el vicio del 
formalismo jurídico, ya que congelan el significado de la regla, muchos de los casos se resolverían de una manera ciega, presentándose lo que se podría denominar: "ceguera jurídica", es un formalismo dogmático, rígido, que trata de encasillar las acciones de los seres humanos como si estas fueran iguales. Las acciones de los seres humanos no funcionan como funciona la lógica, ya lo decía Wittgenstein en uno de los aforismos del Tractatus (1979): "En la lógica no hay sorpresas" y en otro aforismo decía: "la lógica no tiene nada que ver con el mundo". Por estas y otras razones, Hart concibe la tesis del escepticismo de las reglas, es decir, en el fondo es el escepticismo a la teoría pura de derecho y a las distintas teorías formalistas.

Estamos en dos continentes diferentes: el de la lógica, e implícitamente el de la Teoría pura en el Derecho de Kelsen, y en el de la pragmática jurídica que encontramos en la concepción de Hart. Estamos en un campo cerrado, dogmático, absoluto: el campo del cielo o de Dios; y en un campo abierto, inseguro, crítico, y relativo: un campo terrenal o del hombre y del legislador terrenal. De este modo, el jurista se parece más al Dios que concibe Newton que es intramundano y a cada momento le da la cuerda al reloj que es este mundo; digamos que el reloj del formalismo es automático, y el reloj de la pragmática es de cuerda: nos movemos entre un universo cerrado y un universo infinito. Este último universo de textura abierta es el que concibe Hart en el que funciona el sistema jurídico.

Es el momento de señalar que la noción de "textura abierta" se le debe a Waisman (1976), quien la concibe en un escrito que título "Verificabilidad". Allí dice:

"La incapacidad de los fenomenistas para traducir un enunciado sobre un objeto material a términos de datos sensibles no es, como se ha insinuado, debida a la pobreza de nuestro lenguaje, que carece de vocabulario para describir todos los pequeños detalles de la experiencia sensible, ni se debe tampoco a todas las dificultades inherentes a la producción de una combinación infinita de enunciados sobre datos sensibles, aunque todo esto puede contribuir a ella. Se debe principalmente a un factor que, aunque es muy importante y en realidad bastante obvio, nunca ha sido notado que yo sepa: la 'textura abierta' de la mayoría de nuestros conceptos empíricos" (Waisman, 1976, p. 59$60)$.

Se observa la limitación que se tiene para traducir las distintas características de un objeto material a datos sensibles o percepciones, una cosa es el objeto y otra la percepción, de tal manera que es imposible que la percepción pueda captar todas las características que en sí mismos tienen los objetos. Esto se debe a que la mayoría de los conceptos empíricos poseen una textura abierta, lo cual significa que sus notas son infinitas y que nosotros con el concepto empírico solo abarcamos un número limitado de esas notas. El objeto de la percepción es abierto, mientras que el concepto empírico que tenemos es cerrado, lo cual lleva a Waisman a concebir la "textura abierta" de los conceptos empíricos. Sucede con mucha frecuencia, especialmente, cuando confiamos el conocimiento al dato de los sentidos, los cuales en un determinado momento tienen la seguridad de que han percibido un objeto determinado y al momento siguiente se dan cuenta de que ese objeto que habían percibido era otro objeto, u otro individuo, o que según la última percepción ha surgido una especie nueva de individuos. Como la percepción no es completa, se podría afirmar que la percepción es $N$; y que de las propiedades de los objetos son siempre, y siguiendo la fórmula de la inducción matemática, equivalentes a $N+1$, dice Waisman: "El hecho 
de que, en muchos casos, no haya tal verificación conclusiva, se relaciona con que la mayoría de nuestros conceptos empíricos no están delimitados en todas nuestras direcciones posibles" (Waisman, 1976, p. 61). Lo que nos lleva a afirmar que inclusive tampoco es posible tener en la ciencia conocimientos concluyentes o definitivos, no tenemos definiciones exactas, ya que nunca podemos excluir el surgimiento de una situación imprevista, lo que nos llevaría a tener que cambiar nuestro conocimiento inicial, dice Waisman: "Por mucho que tratemos, ningún concepto está delimitado de modo que no quepa ninguna duda" (Waisman,(1976) 1945, p. 61). Esto significa que siempre nos movemos como si las situaciones fueran las mismas, en la misma dirección, como si no hubieran altibajos u otras direcciones: pensamos que de la misma manera como hemos percibido los objetos pasados los vamos a percibir en un futuro. Por ello, según Waisman, no es posible definir un concepto con una precisión absoluta que quede vacunado contra toda posibilidad de dudas, contra toda posibilidad de "textura abierta".

Precisamente en este momento entra Waisman a diferenciar entre la noción de "vaguedad" y la noción de la "textura abierta". Una palabra que se usa de un modo oscilante como "montón", "rosa", "calvo", "pobreza" son palabras vagas, realmente el significado oscila de tal modo que no sabemos cuál es el número de pelos que le hacen falta a una persona para poder afirmar de esta que "es calva". No sucede lo mismo con el término "oro", sabemos su significación, ya que su uso no es vago, pero posee una textura abierta, ya que no tenemos un conocimiento último, lo cual nos puede conducir a muchas dudas. Hay que afirmar que la textura abierta es la posibilidad para la vaguedad, pero que es muy diferente a la vaguedad (Gómez, 2004).

Afirmemos con Waisman que una de las características de los conceptos empíricos es la imposibilidad de la verificación concluyente, dice: "Por lo tanto, la ausencia de una verificación conclusiva se debe directamente a la textura abierta de los términos en cuestión" (Waisman 62). Esto tiene una enorme consecuencia para los fenomenistas, quienes han intentado traducir lo que se entiende por la expresión de un objeto material al lenguaje de la experiencia sensible, pero estos intentos de traducción han sido fallidos. La imposibilidad de traducción se debe a la posibilidad abierta e infinita que tienen los objetos materiales y ante los cuales el lenguaje es muy limitado para realizar una descripción completa. Por ejemplo, tenemos un objeto material $P$ del cual realizamos diferentes descripciones que podemos representar por $a 1+b 1+c+1 \ldots$, y así sucesivamente ad infinitum, además podríamos hacer descripciones más precisas y seguir analizando cada una de las cualidades, por ejemplo, tenemos a1 que puede ser analizado: (a1)1 + (a1)2 + (a1)3... y así sucesivamente. Esto significa que nunca es posible tener la descripción completa, ya que esta siempre posee una textura abierta, o muchas veces puede suceder algo inesperado que se sale del cauce normal de los acontecimientos. A esta imposibilidad de hacer una descripción completa la denomina Waisman: "La deficiencia esencial de una descripción empírica".

La deficiencia esencial de una descripción empírica permite siempre agregar detalles de una nueva descripción, prologándose hacía un horizonte abierto, está en contraposición de la descripción completa como sucede en la matemática y en la geometría, lo mismo que en la lógica o en aquellos conocimientos que poseen en sí misma una demostración. Son saberes de la demostración, como sucede con la descripción de un juego, por ejemplo, el ajedrez, o como lo dijo Leibniz que algo real es siempre inagotable en sus propiedades y es una verdadera imagen de la mente infinita. Mientras en las ciencias formales se puede agotar su descripción, ya que podemos afirmar que su conocimiento es cerrado, nunca podemos agotar la descripción de un objeto material o de una situación: siempre puede suceder algo imprevisto. En la "textura abierta" 
no se dispone de un criterio que nos permita incluir o excluir todos los casos, ya que es imposible prever todos los casos. No se pueden agotar todas las descripciones, ni registrar en fórmulas todas las cualidades de los objetos materiales, siendo una enfermedad de los lenguajes naturales y no de los lenguajes formales.

La concepción de Waisman puso en duda la verificabilidad y supuso una crítica a la concepción fundacionista del empirismo verificacionista. Pero lo más importante que debemos destacar, es la importancia que Hart le dio a la noción de "textura abierta" concebida por Waisman y su aplicación a un sistema jurídico.

A modo de resumen, digamos que en nuestro recorrido sobre esta noción desarrollamos la noción de "textura abierta". Primero, Hart concibe que en un sistema jurídico ocupan un lugar de importancia los términos jurídicos que poseen esta característica, análisis que estuvo seguido de la aplicación de la textura abierta a los términos generales y después a los conceptos empíricos. Segundo, mostramos con Waisman que los conceptos empíricos están sometidos por esta noción u obstáculo para encontrar un conocimiento perfecto, lo mismo sucede con los términos generales; más aún podemos afirmar que "la textura abierta" hace parte de modo esencial del orden jurídico. A modo de esquematizar esto, podemos decir que la textura abierta está presente en: las ciencias empíricas, en los términos generales, en los términos jurídicos, en la moral. Caminamos, entonces, en un campo resbaladizo cada vez más, que va desde: lo empírico, lo racional y lo razonable: ciencias naturales, ciencias formales, y ciencia jurídica y moral.

Para finalizar, debo decir que la verdad se abre a partir de una verdad cerrada a una verdad cada vez más abierta: se pasa de las ciencias matemáticas a las empíricas hasta llegar a la ciencia moral y política. Es el camino que sigue Hume en los diferentes libros del Tratado, se pasa de relaciones de ideas a cuestiones de hecho y posteriormente a acciones morales. Es decir, nos encontramos a nivel de tres razones: razón matemática, razón empírica, y razón moral o política. Lo cual nos indica que simplemente somos seres humanos. Esto nos muestra que no siempre somos seres racionales, y quizás muchas de nuestras decisiones se fundamentan en lo irracional. Se pasa del universo cerrado de las ciencias matemáticas y lógicas, posteriormente se pasa por a través de la ciencias empíricas para llegar por último a las ciencias de la naturaleza humana o ciencias del hombre como las denominó Hume en distintas partes de su obra. Es decir, el camino va del universo cerrado de las matemáticas al universo abierto o universo infinito de la naturaleza humana, nunca se sabría la verdad final.

\section{REFERENCIAS}

- Brown, J. (1965). Técnicas de persuasión. Buenos Aires: Editorial Paidós.

- Descartes, R. (1903a). Discurso del método. En Obras completas. París: Editorial Hachette.

- (1903b). Reglas para la dirección del espíritu. Regla III. En Obras completas. París: Editorial Hachette.

- Descartes, R. (2004). Principios de la Filosofía. Madrid: Alianza Editorial.

- Gómez, A. L. (2004). La importancia de las nociones confusas. Cali: Editorial Universidad del Valle.

- Hart, H. L. A. (2004). El Concepto de Derecho. Buenos Aires: Editorial Abeledo Perrot. 
- Hudson, W. D. (Ed.). (1969). The is-ought question. London: Edit Macmillan.

- Hume, D. (1977). Tratado de la naturaleza humana. Madrid: Editora Nacional.

- _ (2003). Investigación sobre la moral. Buenos Aires: Editorial Losada.

- Kneale, W. M. (1980). El desarrollo de la lógica. Madrid: Editorial Tecnos.

- Lukasiewics, J. (1977). La silogística de Aristóteles. Madrid: Editorial Tecnos.

- Moore, G. E. (1997). Principia Ethica. México: Editorial UNAM.

- Platón. (1969). El Fedro. Madrid: Ediciones Guadarrama.

- Porphyry, B., Abelard, D. S., y Ockham. (1984). Five texts on the medieval problem of universals. Cambridge: Hackett Publishing Company.

- Putnam, H. (2004). El desplome de la dicotomía hecho-valor y otros ensayos. Barcelona: Paidós Básico.

- Waisman, F. (1976). Verificabilidad. En La teoría del significado. México: Fondo de Cultura Económica.

- Wittgenstein, L. (1979) Tractatus lógico -Philosophicus. Madrid: Alianza Universidad.

1. Universidad de Caldas. Alejandro.patino@ucaldas.edu.co. Profesor Titular Departamento de Filosofía.Universidad de Caldas.

2. La definición por género y especie fue criticada por Descartes en la segunda Meditación Metafísica cuando concibe que estas dos nociones conducen a una regretio ad infinitum. Por ello, propone reducir la cuestión de Hombre a las partes más simples, en este caso a su naturaleza simple como son la figura, la extensión, la forma. El análisis nos conduce a las partes evidentes e indivisibles. Esto evita cualquier tipo de regretio ad infinitum.

3. Las críticas al silogismo las encontramos en diferentes partes de las obras de ambos filósofos. Estas críticas fueron normales en la época moderna y se encuentran tanto en los racionalistas como en los empiristas.

4. Estudios muy serios sobre el silogismo los encontramos en Kneale (1980), El desarrollo de la lógica, y en Lukasiewics (1977), La silogística de Aristóteles.

5. Debemos recordar que la axiomática de Euclides la expuso en su obra Los Elementos. Una demostración axiomática consta de: términos primitivos, axiomas, postulados y demostraciones.

6. Recordemos que se presentan dos momentos: el regresivo y el progresivo. En el primero, hay que eliminar los prejuicios y dejar la razón en estado puro, una vez en este estado, se pasa al estado progresivo. Es decir, se pasa de una razón en estado de ignorancia a una razón con conocimiento.

7. Descartes. RDE V. En esta regla Descartes relaciona la segunda regla con la tercera que tiene que ver con el orden.

8. Cfr. Porphyry et al. (1984), Five texts on the medieval problem of universals.

9. De ahí que en la deducción sea necesario la presencia de la razón y de la memoria, siendo la deducción una sumatoria de intuiciones intelectuales.

10. Moore (1997), Principia Ethica. Esta falacia la desarrolló en los dos primeros capítulos, criticando a quienes reducen las cuestiones de valor a cuestiones de hecho como si tuvieran el mismo estatus que los fenómenos naturales. Por ello, Moore se ve en la necesidad de concebir a la intuición como operación que 
percibe todas las acciones de valoración ética. Es esta la razón por la cual se identifica a Moore como un intuicionista.

11. Hume (2003), Investigación sobre la moral. Debemos recordar que esta obra fue publicada por Hume en 1751 y que corresponde a una elaboración más madura de las tesis que desarrolló en el tercer libro del Tratado de la naturaleza humana, obra a la que nos vamos a referir con frecuencia en las páginas siguientes.

12. Tesis que influye en los positivista-lógicos como Carnap y Ayer. Con Base en esta tesis empieza Putnam (2004) el primer capítulo de su libro El desplome de la dicotomía hecho, valor y otros ensayos. Se debe tener en cuenta que Putnam está en contra de quienes defienden esta dicotomía lo mismo que la dicotomía analítico-sintético.

13. Esta polémica la encontramos en el libro editado por Hudson (1969), The is-ought question. En este libro aparecen algunos autores que defienden la imposibilidad del paso del "es" al "debe" y otros que la conciben.

14. Cfr. Hume (2003, p. 169).

15. Cfr. Hume (2003), Sección primera "De los principios generales de la moral" y primer apéndice a este libro, "Acerca de sentimiento moral". 